\title{
Extraction of urban features in Strasbourg, France: Comparison of two fusion algorithms for Quickbird MS and Pan data
}

\author{
Christiane Weber, Thierry Ranchin, Member, IEEE, Anne Puissant and Aziz Serradj
}

\begin{abstract}
Urban areas might be defined as a complex and dynamic system that needs specific and reliable products to be observed and managed. Very High Resolution (VHR) satellite, such as Quickbird image, provides sub-meter spatial resolution images in the panchromatic mode and multispectral images Hence, the idea of fusing synthetic multispectral images with a highest spatial resolution, enable the creation of usable products for urban planning and operational purposes. Such kind of techniques provides imagery products that need to be assessed in term of quality and reliability towards urban practises and purposes. This paper compares quantitatively and qualitatively two fusion methods over the Strasbourg area (France). It defines a more complete set of parameters for the use of fusion in urban areas studies and leads to quality conclusions regarding global and local assessments.
\end{abstract}

Index Terms - Fusion methods, urban features, comparison

\section{INTRODUCTION}

$\mathrm{T}$ he spatial resolution plays a very important role in the studies of urban areas by means of satellite images taken in the optical domain. Most of the space-borne sensors presently operated provide data sets comprising multispectral images at a low spatial resolution and images at a higher spatial resolution but with a lower spectral content. Planned earth observation systems adopted such a principle. The Very High Resolution (VHR) satellite such as Quickbird, provides a sub-

Manuscript received March 1, 2003.

C.H. Weber is with the French National Scientific Research Center (CNRS), UMR 7011 CNRS/ULP Strasbourg (phone: +33 390-240-974; fax: +33 390-240-950; e-mail: christiane.weber@lorraine.u-strasbg.fr).

T. Ranchin is with the Ecole des Mines de Paris, BP 207, 06904 Sophia Antipolis, France (phone: +33-493957460; fax: +33-493957535; e-mail: thierry,ranchin@ensmp.fr).

A. Puissant is with the French National Scientific Research Center (CNRS), UMR 7011 CNRS/ULP Strasbourg (phone: +33 390-240-9 60; fax: +33 390-240-950; e-mail: anne.puissant@lorraine.u-strasbg.fr).

A. Serradj is with the French National Scientific Research Center (CNRS), UMR 7011 CNRS/ULP Strasbourg (phone: +33 390-240-9 68; fax: +33390-240-950; e-mail: anne.puissant@lorraine.u-strasbg.fr). meter spatial resolution images in the panchromatic mode and four multispectral images with a spatial resolution four times coarser than the panchromatic band. This VHR data gives access to very precise information for urban analysis.

Compared to natural landscapes, urban areas are composed of diverse materials (concrete, asphalt, metal, glass, water, grass) organised into regularly shaped land parcels (Welch, 1982; Jensen and Cowen, 1999), and characterised by urban objects such as buildings, houses, transportation networks, utilities, parks. Hence, urban heterogeneity requires an adapted, very high, spatial and spectral resolution (Puissant and Weber, 2002).

The multiplication of details will lead to develop new processing method combining geometric and radiometric characteristics of urban features. Indeed, these latter influence the ability to analyse different types of urban structures. The geometric criteria include surface, shapes and organisation of the urban objects. The radiometric criteria include specific reflectance of the constitutive elements of the urban objects.

Hence, the idea of having synthetic multispectral images with a highest spatial resolution leads to developments in image fusion. The benefits of having such a kind of images have been clearly demonstrated by a number of papers (see $e . g$. Couloigner et al. 1997, 1998a, b; Raptis et al. 1998; Vaiopoulos et al. 2001; Wald and Ranchin 2001).

This paper focuses on a comparison analysis of two fusion methods: the implementation of the ARSIS concept (Wald and Ranchin 2001), and Correlation (Weber et al., 1996). This comparison will achieve information on the best fusion algorithm for different channels characteristics and various urban elements. Indeed urban features may require different processing methods according to whether they are built-up, or vegetation, or concrete elements. From a list of urban elements precisely described for the analysis of an urban area, some have been selected in order to allow comparison and control of the fusion methods results. After the presentation of the urban context, the fusion methods are briefly described in the second part. The quality assessment, global and local, is detailed in the third part and measurements have been realized on homologous elements (geometric measures, variance, image correlation and so on). Finally some concluding remarks have been drawn on the difference between the two algorithms and on the benefits of their use in urban studies. 


\section{DATA USED AND STUDIED AREA}

The Quickbird images (acquired in May 2002) have a spatial resolution of $2.8 \mathrm{~m}$ in four bands (B0: $0.45-0.52 \mu \mathrm{m}$, B1: 0.52-.60 $\mu \mathrm{m}, \mathrm{B} 2: 63-0.69 \mu \mathrm{m}$ and B3: $0.76-.90 \mu \mathrm{m})$ and the panchromatic mode has a spatial resolution of $0.7 \mathrm{~m}(\mathrm{P}$ : $0.45-0.90 \mu \mathrm{m})$. The full dynamic range of images $(11 \mathrm{bits})$ coded in 16 bits is used to apply in the two fusion algorithms.

The selected urban elements used in the comparative analysis cover the urban area of Strasbourg (France). This area is representative of the urban structure of western cities and is characterised by many different objects that exhibit a diverse range of spectral reflectance values. The urban features regroup the basic object referring to the land cover (trees, grass, building, road -asphalt, water). A shadow theme has been added, as it is largely present in such resolution.

\section{ANALYSIS METHOD FOR URBAN AREAS}

Urban analysis is a complex domain due to multiple interactions between the social, political and environmental spheres. The field of urban development is thus composed of different types of applications: firstly daily management of the area (network, facilities and green spaces), secondly, urban planning (operational planning, impact study, regulatory documentation) and finally urban prospective (development scenario). All these applications require access to reliable upto-date data and a good knowledge of land cover and its evolution.

Currently the domain of planning and urban analysis is characterized by multi-criteria decision-making, which is typically very sensitive to context (Mesev and al., 2000). These requirements lead end-users to combine multisource information at various scales (French Equipment Ministry, 1999). In this context, Earth observation data (aerial or satellite) is a potential source of information, but seldom used by urban planners (SCOT, 1997). Results of surveys of potential end-user requirements (Puissant and Weber, 2001) have shown that currently the main applications in urban areas concern the tactical and technical levels for which 1:200 to 1:10,000 scales are needed (Table I).

TABLE I

END-USER APPLICATIONS AND IMAGE DATA

\begin{tabular}{|c|c|c|c|}
\hline \multicolumn{4}{|c|}{ END-USER APPLICATIONS AND IMAGE DATA } \\
\hline Apglinwiras & Nastes & Rroluline & 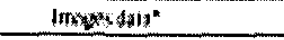 \\
\hline 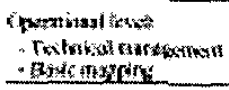 & 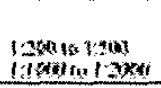 & $\begin{array}{l}211 \\
\text { Hitgen }\end{array}$ & 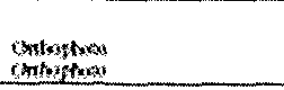 \\
\hline $\begin{array}{l}\text { Totidul krit } \\
\text { pluation }\end{array}$ & 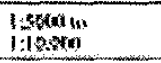 & $\begin{array}{l}\sin 6 \ln 3 \\
\log \sin \end{array}$ & 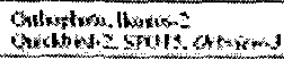 \\
\hline 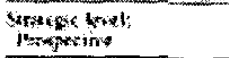 & $\begin{array}{l}\text { lithentas } \\
\text { li intions }\end{array}$ & 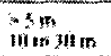 & 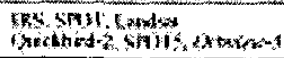 \\
\hline
\end{tabular}

For these applications, the most useful source of image data is aerial photography. In fact, sub-meter resolution orthophotos are the only image used (Puissant and Weber, 2001). As satellite images did not achieved better resolutions than the $5.8 \mathrm{~m}$ of the IRS sensor (except Ikonos and Quickbird which is too expensive for most planning agencies), these data were only used for $1: 25,000$ to $1: 1,000,000$ scales applications. Indeed, for this old generation of so-called "High Resolution" satellite sensor (with a spatial resolution of more than $5 \mathrm{~m}$ ), basic urban objects are commonly smaller than the size of the image pixels. For the new generation, the so-called "Very High Resolution" of satellite sensors (Fritz, 1996), which produce digital image data with a spatial resolution of 0.61 to $5 \mathrm{~m}$ (Ikonos launched in 1999, Quickbird in 2001, and SPOT 5 in 2002), urban objects are typically larger than the image pixels. These new sensors with resolutions similar to aerial photography, can identify urban objects. These resolutions enable the analysis of the spatial pattern of cities at scales between 1:10,000 and 1:25,000, typical of projects dealing with urban planning (Donnay and al., 2000). It is anticipated that relevant imagery products will be used, if their performance has been assessed. Two main milestones have to be successfully overcomed: (1) the assessment of identification capacity through satellite imagery products for urban analysis and (2) the enhancement of classification methods enabling the understanding of the studied urban space. The first point requires precise and sharp location, the second knowledgebased inputs for classification methods. Regarding the first step fused documents might provide such confidence in satellite imagery products if spectral and geometric qualities are preserved.

\section{FUSION METHODS FOR URBAN AREAS ANALYSIS}

In this framework of analysis of urban areas, it is then of interest to evaluate the influence of two algorithms of data fusion on the results. The first one is derived from the ARSIS concept (Ranchin and Wald, 2000). This algorithm makes use of a multiscale approach and of high quality transformation of the information of the panchromatic image of Quickbird. The used algorithm for the implementation of the ARSIS concept is the so-called UWT-M2 (Ranchin and Wald, 2003). It makes use of an undecimated wavelet transform and to the Inter Modality Model called M2.

The second one is the correlation coefficients method. It is based on the correlation coefficient between the panchromatic band and the multispectral band concerned (Weber et al., 1996). The algorithm used for Quickbird images is:

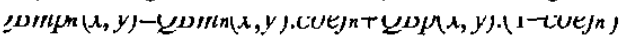

where:

$$
\begin{aligned}
& 2 B m b_{k}(x . v)=\begin{array}{l}
\text { Pan-sharpened pixel for band } \mathrm{n} \\
\text { Digital Number for multispectral Quickbird }
\end{array} \\
& \begin{array}{l}
\text { band } \mathrm{n} \\
\text { Digital Number for panchromatic Quickbird } \\
\text { band }
\end{array} \\
& \text { Correlation coefficient between the panchromatic } \\
& \text { and the multispectral band } \mathrm{n}
\end{aligned}
$$

Several equations have been tested for the correlation method (Figure 1). The large range of panchromatic values of the Quickbird image $(0.45-0.90 \mu \mathrm{m})$ and its strong correlation with the near-infrared band has led to used the inverse relation in order to reduce the importance of the 
panchromatic (2).

$$
Q B m p_{n}(x, y)=\left(Q B_{n}(x, y) \cdot\left(l-\operatorname{coef}_{n}\right)\right)+\left(Q B p(x, y) \cdot\left(\operatorname{coef}_{n}\right)\right)
$$

where:

$Q B m p_{n}(x, y)=$ Pan sharpened pixel for band $\mathrm{n}$

$Q B m_{n}(x, y)=$ Digital Number for multispectral Quickbird band $n$

$\operatorname{coef}_{n}=$ Correlation coefficient between the panchromatic and the multispectral band $n$

The result is compared to the UWT-M2 product (Figure 2).
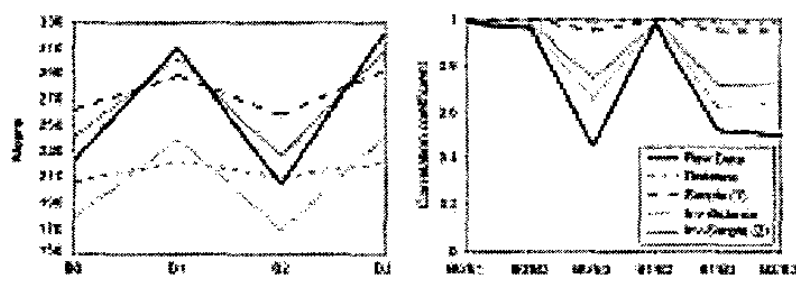

Fig. 1. Test on fusion by correlation

\section{QUANTITATIVE ASSESSMENT OF THE FUSION METHODS.}

The two images results have been introduced in a comparison protocol to assess their quality quantitatively and qualitatively regarding the original multipsectral bands resampled at the spatial resolution of the pan-sharpened image and their utility to answer to the urban analysis requirements. This protocol relying on spectral and geometric criteria is defined into two steps: a global study on a large part of the city and a local study more specifically focused on urban objects.

\section{A. Global quality assessment}

The quantitative analysis of the fusion methods was done according to the protocol defined by Wald et al. (1997). All the computations were made on the full dynamic range of images (11 bits). These fusion products are synthetic images aiming at simulating what a sensor having the same spectral bands but the highest spatial resolution would observe.

The properties of these synthetic images $B^{*}{ }_{\mathrm{h}}$ have been established by Wald et al. (1997):

1) Any synthetic image $B^{*}$ once degraded to its original resolution $l$, should be as identical as possible to the original image $B_{1}$

2) Any synthetic image $B^{*}$ should be as identical as possible to the image $B_{\mathrm{h}}$ that the corresponding sensor would observe with the highest spatial resolution $h$, if existent.

3) The multispectral (or multi-modality) set of synthetic images $B^{*}{ }_{h}$ should be as identical as possible to the multispectral (or multi-modality) set of images $B_{\mathrm{h}}$ that the corresponding sensor would observe with the highest spatial resolution $h$, if existent.

As an illustration, Table II deals with the test of the second property and reports some statistics on the relative discrepancies between the original images $B_{k l}$ and the images $B^{*}{ }_{k l}$. The differences are computed on a pixel basis and one image of differences is obtained per spectral band $k$. From each image of differences, the mean value (bias), standard deviation are computed. These quantities are expressed in percent, relative to the mean radiance value of the original image $B_{k l}$. The ideal value for these parameters is 0 . In addition, the difference between the variance of the original image $B_{k l}$ and that of $B^{*}{ }_{k l}$ is computed. It is expressed in percent, relative to the variance of the original image. Ideally, this value should be zero. The correlation coefficient between the original image $B_{k l}$ and $B_{k l}^{*}$ is also computed. The ideal value is 1 . TABLE II GLobal Quality STATISTICS

\begin{tabular}{|c|c|c|c|c|c|}
\hline & & Bias & $\begin{array}{l}\text { Standard } \\
\text { Dev. }\end{array}$ & $\begin{array}{c}\text { Diff. in } \\
\text { Variance }\end{array}$ & $\begin{array}{l}\text { Correlation } \\
\text { Coeff. }\end{array}$ \\
\hline Blue & $\begin{array}{l}\text { CORRELATION } \\
\text { UWT-M2 }\end{array}$ & $\begin{array}{l}-11 . \overline{3} \\
-0.02\end{array}$ & $\begin{array}{l}29.4 \\
25.4 \\
\end{array}$ & $\begin{array}{r}-7.6 \\
-14.2\end{array}$ & $\begin{array}{l}0.793 \\
0.852\end{array}$ \\
\hline Green & $\begin{array}{l}\text { CORRELATION } \\
\text { UWT-M2 }\end{array}$ & $\begin{array}{l}1.25 \\
0.03\end{array}$ & $\begin{array}{l}34.5 \\
29.9\end{array}$ & $\begin{array}{l}22.8 \\
-19.7\end{array}$ & $\begin{array}{l}0.700 \\
0.860\end{array}$ \\
\hline Red & $\begin{array}{l}\text { CORRELATION } \\
\text { UWT-M2 }\end{array}$ & $\begin{array}{l}-20.7 \\
-0.23\end{array}$ & $\begin{array}{l}42.6 \\
40.1\end{array}$ & $\begin{array}{l}15.9 \\
-17.6\end{array}$ & $\begin{array}{l}0.797 \\
0.848\end{array}$ \\
\hline NIR & $\begin{array}{l}\text { CORRELATTON } \\
\text { UWT-M2 }\end{array}$ & $\begin{array}{c}2.8 \\
-0.14\end{array}$ & $\begin{array}{l}44.8 \\
32.2 \\
\end{array}$ & $\begin{array}{l}17.8 \\
-20.7\end{array}$ & $\begin{array}{l}0.643 \\
0.851\end{array}$ \\
\hline
\end{tabular}

For the UWT-M2 (ARSIS) method, the bias is very small. The correlation method introduces a strong bias in all bands. The standard deviations are comparable for the two methods, but it always lower for the UWT-M2 method. The correlation coefficients are better for the UWT-M2 method than for the Correlation method. For the Correlation method, the most difficult case is the NIR band.

\section{B. Global urban reliability}

The global study gathers all different urban objects located in a urban area. Variance analysis has been run for each channel and a correlation analysis set up. Variance comparisons highlight a large similarity between the original image at $0,7 \mathrm{~m}$ and the ARSIS fused result for the four channels. The correlation method introduces more differences for the blue and the near infrared channels.

More precisely the variance is overestimate in the blue channel and underestimate for the near infrared, and more lightly for the green channel. A variance overestimation comparatively to the original channel shows that the correlation method introduce too much information. Inversely a under-estimation corresponds to a loss of information. Only the red channel (band 3 ) is correctly provided by both fusion methods (Figure 2).
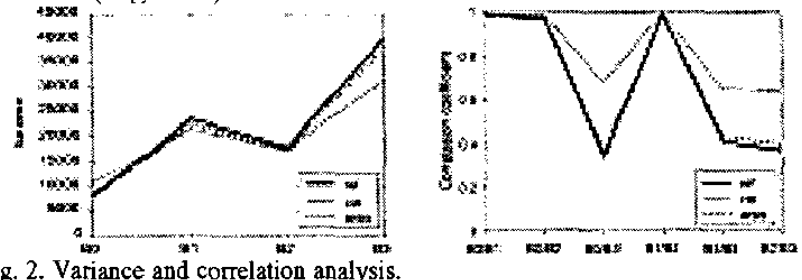

The correlation coefficient analysis between each channel (Figure 2) the less good fused result for the channel 4 obtained by correlation method. The ARSIS method proposes better radiometric quality results than the correlation method. But a detailed analysis shows a good level of information for the channels green and red, less good for the blue and the near 
infrared. A local analysis might confirm these results.

Qualitative assessment of the fusion methods.

The local study has been realised on various selected urban objects according their spatial resolution and spectral characteristics. Three types of buildings' roofs with materials have been chosen (tiles, gravels and metal/steel roofs). Vegetation elements like trees and grass, some concrete surfaces (roads) and water surfaces have also been selected. Shadow has also been pointed out, as it constitutes a permanent element in urban area.

The local analysis is based: (1) on the comparison of radiometric cross-sections and (2) on the behaviour of urban objects through the different bands. For each object category the mean point (mean of five object) is represented in dispersion graphs.

Four radiometric cross-sections have been realised over a set of urban objects in order to assess all of the selected urban objects and to check the constancy behaviour of these elements in the fourth bands. Figures 3 and 4 represent two of these cross-sections. They cross a distance of $140 \mathrm{~m}$ (200 pixels) and are composed about ten elements gathering a maximum of five categories of urban elements categories.

Firstly radiometric cross-sections are studied using statistical results for each urban object on the four crosssections and for each band (reference images, pan-sharpened image by ARSIS and by Correlation). The values of the pansharpened images for the crossed urban elements might be above, under or equal to the reference data. The statistics on these three curves allow to assess qualitatively the quality of the spectral behaviour for the various elements whatever the channel.

The first results show that ARSIS method provides a good quality of image equal to the original for all the selected elements. The correlation method provides good results only for three categories: the roads, the water surfaces and the shadow.

Secondly, on the one hand, the most recurrent behaviour for each type of urban objects, channel by channel, is identified to complete the quality assessment of the spectral response; and on the other hand, the means values coming from the pansharpened images (estimated values) are compared to the reference values (actual values) to complete this analysis (Figure 5).

The correlation method tends to overestimate different types of elements: grass, tree in channels 0, 1, 2. And it underestimates them in channel 3. Water surfaces and shadow are inversely taken into account. For the steel and the gravel roofs and the roads, an overestimation is calculated in channels 0 and 1 , and they are correctly provided in channels 2 and 3. ARSIS method provides better results in general. All categories are well rendered in channel $0,2,3$. But an overestimation for vegetation and water surface can be discerned in channel 3.

\section{CONCLUSION}

The issue of Very High Resolution imagery products has been tackled in this paper. Table I clearly shows the limits and benefits of these products for urban studies. In order to improve the usefulness of these products in urban studies, the use of fusion algorithms has been evaluated. Various methods of fusion might be applied on very high satellite imagery products such as Quickbird images in order to provide useful and reliable products for urban studies. In this paper two of them, the UWT-M2 method based on the ARSIS concept and the Correlation method, were compared within the aim of providing useful information on urban areas.

Qualitative and quantitative parameters have been combined in a unique protocol. This protocol combines global and local analysis for specific urban features, and allows the evaluation of the fused product for urban studies.

\section{REFERENCES}

[1] Couloigner I., Ranchin T., Valtonen V. P., Wald L., 1997. Appont de SPOT 5 à la cartographie urbaine. Bulletin de la Société Française de Photogrammétrie et Télédétection, 145, 1, 33-38, 1997.

[2] Couloigner I., Ranchin T. and L. Wald, 1998a. Benefit of data fusion to urban roads mapping. In Proceedings of the second conference "Fusion of Earth data: merging point measurements, raster maps and remotely sensed images", Sophia Antipolis, France, January 28-30, 1998, T. Ranchin and L. Wald Editors, published by SEE/URISCA, Nice, France, pp. 183-190.

[3] Couloigner I., Ranchin T., Valtonen V.P. and Wald L., 1998b. Benefit of the future SPOT 5 and of data fusion to urban mapping. International Journal of Remote Sensing, 19(8):1519-1532.

[4] Jensen J.R. and Cowen D.C., 1999. Remote sensing of urban/suburban infrastructure and socio-economic attributes. Photogrammetric Engineering and Remote Sensing, 65, pp. 611-622.

[5] Mesev V.B., Gorte P., Longley M.A, 2000. Modified maximumlikelihood classification algorithms and their application to urban remote sensing, in Remote Sensing and Urban Analysis, GISDATA 9, Ed. Taylor \& Francis, Great Britain, pp. 71-94.

[6] Puissant A. and Weber C., 2002. The utility of very high spatial resolution images to identify urban objects. Geocarto International, vol. 17, $n^{\circ} 1$, pp. 31-41.

[7] Puissant A., Hirsch J., and Weber C., 2002. The utility of texture analysis to improve per pixel classification for high spatial resolution imagery, International Journal of Remote Sensing (in press).

[8] Ranchin T., Wald L., 2000. Fusion of high spatial and spectral resolution images: the ARSIS concept and its implementation. Photogrammetric Engineering \& Remote Sensing, 66, 1, pp. 49-61.

[9] Raptis V. S., Vaughan R. A., Ranchin T., Wald L., 1998. Assessment of different data fusion methods for the classification of an urban environment. In Proceedings of the second conference "Fusion of Earth data: merging point measurements, raster maps and remotely sensed images", Sophia Antipolis, France, January 28-30, 1998, T. Ranchin and L. Wald Editors, published by SEE/URISCA, Nice, France, pp. 167-182.

[10] Vaiopoulos D., Nikolakopoulos K., Skianis G., 2001. A comparative study of resolution merge techniques and their efficiency in processing image of uban areas. In Proceedings of the IEEE/ISPRS joint Workshop on Remote Sensing and Data Fusion over Urban Areas, Roma, Italy, November, 8-9th, 2001, pp. 270-274.

[11] Wald L., Ranchin T., 2001. Data fusion for a better knowledge of uban areas. In Proceedings of the IEEE/SPRS joint Workshop on Remote Sensing and Data Fusion over Urban Areas, Roma, Italy, November, 8-9th, 2001, pp. 127-132.

[12] Weber C., Hirsch J. et Serradj A., 1996, Cartographie d'une forêt spécifique : la forêt alluviale de la Robertsau. in Télédétection et Cartographie. Ed. AUPELF-UREF. Presses de l'Université de Québec. p 275-282.

[13] Welch, R, 1982. Spatial resolution requirements for urban studies. International Journal of Remote Sensing, 3, pp. 139-146.

[14] Donnay J.P., Barnsley M.J., Longley P.A., 2000. Remote sensing and urban analysis, in Remote Sensing and Urban Analysis, GISDATA 9, Ed. Taylor \& Francis, Great Britain, pp. 3-18.

Dr. Christiane H. Weber has got her thesis (Phd) in 1982. The subject was a comparative analysis between Rhine valley cities : Strasbourg and Mulhouse (F), Bale (CH), Fribourg (G). She is since 1985, full time researcher at the Centre National de la Recherche Scientifique (CNRS) in France and works in the IMAGE et VILLE research unit (UMR 7011 CNRS/ULP) at the geographyt department of the Louis Pasteur University (Strasbourg). She has a wide experience on uban and spatial analysis, remote sensing applications 
particularly in uban field, and in Geographic Information System. She is a member of the remote sensing research group in the CNRS laboratory (UMR $7011)$ and works with several students on european sites. She gives lectures for graduated students in remote sensing and GIS in the geography department, for professionals and educational courses. She has published more than 90 publications, communications in international symposia, or articles in journals with peer review committees in the field of Geography, remote sensing of the Earth system and GIS. Dr. Christiane H. Weber is involved in different Remote sensing and GIS networks set up in Europe (ESF, AGILE, SIGMA-CASSINI). Her domain of expertise is mainly in urban geography and planning. She is involved in several national or international research programs. She took the direction of the laboratory in 2000

Thierry Ranchin (M'01) a $\mathrm{PhD}$ degree from the university of Nice Sophia Antipolis, France in the field of applied mathematics.

After a post-doctoral fellow in a company in Tromso, Norway, he joined the remote sensing group of Ecole des Mines de Paris in the fall of 1994. He was an invited scientist from the University of Jena, Germany in 1998. He has a patent about sensor fusion and more than 90 publications, communications in international symposia, or articles in journals with peer review committees in the field of remote sensing of the Earth system and in the field of image processing.

Dr. Ranchin received the Autometrics Award in 1998 and the Erdas Award in 2001 for articles on data fusion.

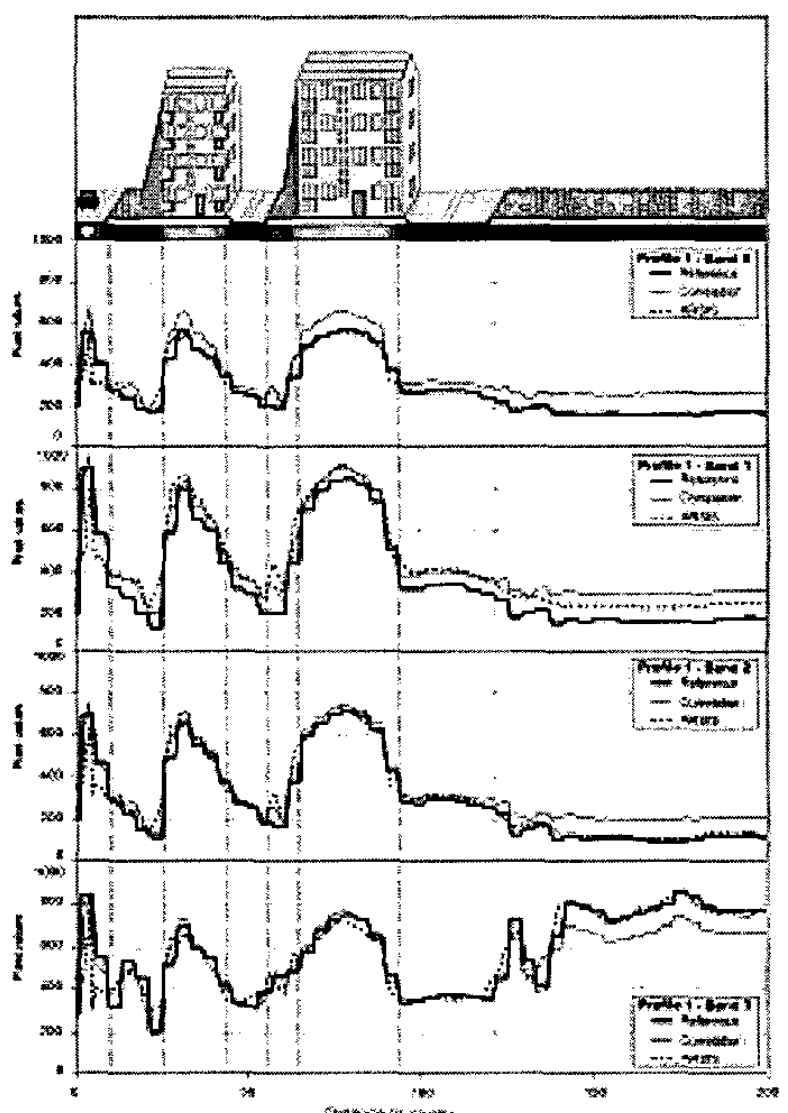

Fig. 3. Radiometric cross-section 1 .

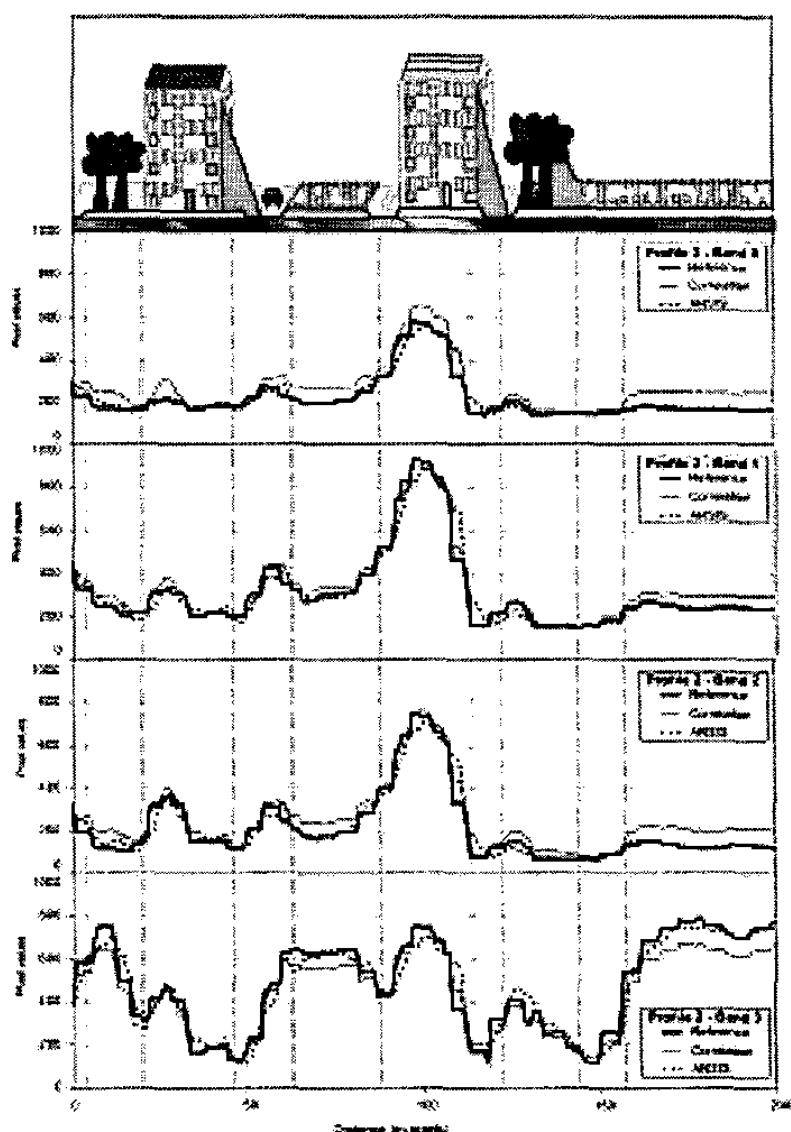

Fig. 4. Radiometric cross-section 2.
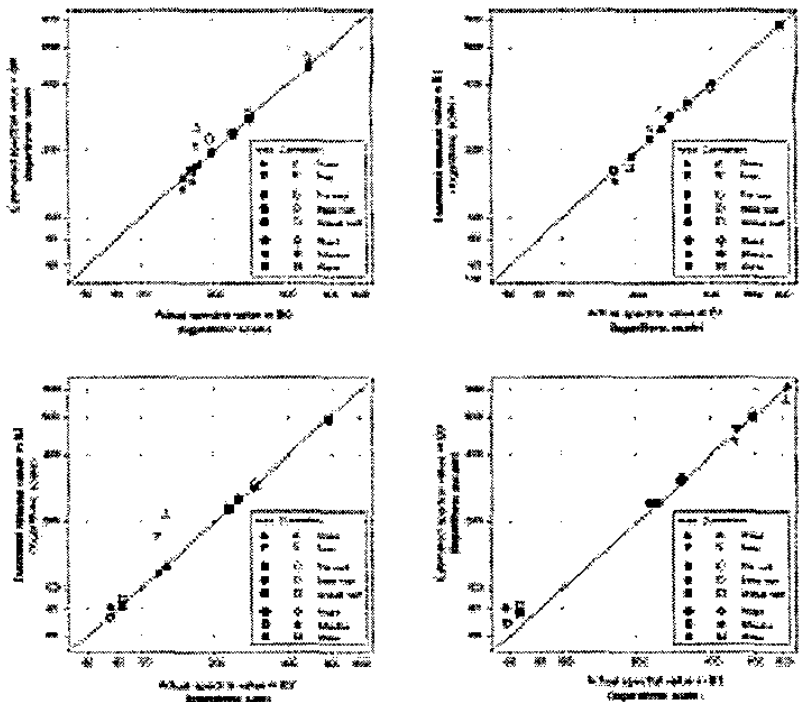

Fig. 5. Comparison between actual and estimated spectral values. 